\title{
The strained state cosmology
}

\section{Angelo Tartaglia}

Politecnico di Torino and INFN, Torino, Italy

E-mail: angelo.tartagliadpolito.it

This talk presents an interpretation of the cosmological constant and of the dark energy, in terms of deformation energy density of space-time. The latter is treated as an elastic medium, whose Lagrangian density is built of terms proportional to the lowest (in practice the second) order scalars associated with the strain tensor, i.e. the difference between the actual and the Euclidean metric tensors, and its first and second order derivatives. The new Einstein equations contain an additional 'source' corresponding to the deformation energy density of space-time. Global symmetries may be induced by texture defects in the manifold. Solutions endowed with Robertson-Walker, Schwarzschild and Kerr symmetries are discussed. The difference from massive gravity theories is evidenced.

Frontiers of Fundamental Physics 14

15-18 July 2014

Aix Marseille University (AMU) Saint-Charles Campus, Marseille, France 


\section{Introduction}

Our knowledge of the universe at the largest scale suffers from a number of open problems. On one side general relativistic cosmology provides a reasonably simple scheme that describes quite well an asymptotically expanding universe made of an homogeneous and isotropic dust of galaxies; on the other side, however, the observation of the sky, and in particular of distant type Ia supernovae, suggests that there is something uniformly permeating the universe, which 'pushes', driving an accelerated expansion of space. The above something has apparently strange properties: it does not gravitate, which means that it does not clump, but what is really surprising, while space expands it does not get diluted. The name commonly used for this strange ingredient is dark energy. Formally it is easy to account for dark energy in the framework of General Relativity (GR). It is enough to add to the Einstein equations a cosmological constant $\Lambda$. The history of $\Lambda$ is rather peculiar and showing how physics progresses sometimes: initially introduced by Einstein, then rejected almost with shame, finally revived in the 90's of the last century. The point is that this constant 'energy density of the vacuum' we do not know what it is.

Dark energy is not the only mysterious ingredient suggested by the observation of the universe. At the scale of galaxies or of clusters of galaxies or even stars, apparently there is something else missing in order to explain the motion of stars and galaxies under the action of gravity: a missing, otherwise invisible, mass is needed. The 'missing mass' of the 30's is now called dark matter: it by definition gravitates, but we do not know what it is. I shall not touch upon dark matter, but just remark that, as a matter of fact, the standard $\Lambda$ CDM model (where CDM stays for Cold Dark Matter) works quite well in accounting for the observations.

Here I shall resume and outline an interpretation of dark energy in terms of deformation energy of space-time associated with the curvature of the four-dimensional manifold [ [ [ ]]. In other words space-time will be treated as an elastic deformable continuum in four dimensions, extending and applying by analogy what we know about ordinary three-dimensional continua. I shall speak of a Strained State Theory (SST). As we shall see, the result, besides being compliant with our intuition, will account for the accelerated expansion of the universe keeping consistency with the main cosmological tests. Preliminary application of the model to peculiar 'local' symmetries, such as Schwarzschild's and Kerr's, suggests the possibility to avoid the need for dark matter. A mention will also be made to the role of texture defects in the space-time deformable continuum, as possible seeds, if not substitute, for matter aggregations.

\section{The Lagrangian of space-time}

When trying to describe the behaviour of both classical and quantum fields, and in general of systems made of mutually interacting parts, we assume that the evolution is governed by a sort of 'economy' principle that is called the least action (actually extremal action) principle. The evolution parameter, in a classical approach, is time and it is assumed that there is an integral (the action integral) which is minimized (or extremized) along the physical evolution path. In a fourdimensional relativistic framework one may keep the approach of the path integral along histories, using proper time, but it is more appropriate to use the action principle as a selection criterion among different global configurations of the whole four-dimensional manifold. In practice the 
action integral $S$ is written as follows:

$$
S=\int * \mathfrak{L}=\int \mathscr{L} \sqrt{|g|} d^{4} x
$$

In the formula the scalar $* \mathfrak{L}$ is the Hodge conjugate of a 4-form. When writing it explicitly in a given coordinate system, one has a four-integral of a scalar density: $\mathscr{L} \sqrt{|g|}$. Of course $g$ is the determinant of the metric tensor (and is used to build the invariant four-volume element) and $\mathscr{L}$ is the Lagrangian density of the system.

The Lagrangian density of space-time is some scalar function (actually a scalar density) of the Lagrangian coordinates of the system (the state parameters), together with their first derivatives. Beyond this constraint we have only some heuristic criteria: the Lagrangian density should be reasonably simple (as simple as possible?); the number of free parameters should be as low as possible; additional components in the system should contribute additional terms in the Lagrangian... In classical mechanics the Lagrangian density is the difference between the kinetic and the potential energies per unit mass; in GR the Lagrangian density of space-time coincides with the scalar curvature $R$. We know, however, as we have seen in the Introduction, that there is something missing. There are various ways to try and tackle this problem, but most often they consist in manipulating the Lagrangian density adding more 'fields' or introducing non-simple functional dependencies on the relevant scalars. The proposals one can find in the literature are usually driven by the result one wants to obtain, but often have uncertain (or entirely missing) physical interpretation.

Here I present a Lagrangian density modelled on known three-dimensional physics of continua generalized to four dimensions. The scalar curvature $R$ appearing in the Einstein-Hilbert action contains first and (linearly) second order derivatives of the elements of the metric tensor, so being the analog of the kinetic term in the classical Lagrangian. It is reasonable to expect the presence also of a potential energy term depending on the metric elements. On the other hand the spacetime of GR is indeed a physical continuum where interesting physics happens when curvature is present and we know, from our ordinary $3+1$ experience, that introducing curvature in a physical continuum requires work and leads to elastic deformation energy.

\section{The Strained Space-Time}

From the classical elasticity and deformation theory of continua we may introduce, as relevant physical quantity, the strain tensor, $\varepsilon_{\mu v}$ proportional to the difference between the actual metric tensor of the deformed medium, $g_{\mu \nu}$, and the corresponding undeformed metric $E_{\mu \nu}$ :

$$
\varepsilon_{\mu v}=\frac{g_{\mu v}-E_{\mu v}}{2}
$$

The most general reference metric , $E_{\mu \nu}$, is of course flat and is assumed to be Euclidean, even though in relativity the flat tangent space is Minkowskian. The signature flip is attainable with a Wick rotation (one imaginary coordinate) and is discussed in ref. [B]].

The elastic potential energy associated with the strain is readable out of the free energy written in terms of the scalars of $\varepsilon_{\mu v}$ :

$$
\mathscr{F}=\mathscr{F}_{0}+\frac{\lambda}{2}\left(\varepsilon_{\alpha}^{\alpha}\right)^{2}+\mu \varepsilon_{\alpha \beta} \varepsilon^{\beta \alpha}+\ldots
$$


Stopping the development to the second order scalars corresponds to the linear elasticity theory which I am assuming to be valid for space-time. Then, apart from the irrelevant constant $\mathscr{F}_{0}$, the two parameters of the theory correspond to the classical Lamé coefficients $\lambda$ and $\mu$.

Summing up, the Lagrangian density of the universe may be written as:

$$
\mathscr{L}=R-\frac{\lambda}{2}\left(\left(\varepsilon_{\alpha}^{\alpha}\right)^{2}+2 \mu \varepsilon_{\alpha \beta} \varepsilon^{\beta \alpha}\right)+2 \kappa \mathscr{L}_{\text {matter }}
$$

The last term accounts for the presence of matter and is multiplied, as usual, by the coupling constant $\kappa=8 \pi G / c^{4}$. The first couple of terms accounts for geometry or, to say better, for the intrinsic properties of space-time, however one may also look to the second term as to a separate source for curvature, leading to an elastic energy/momentum tensor (even though it is essentially a reaction to curvature).

In the classical theory of elastic continua we know that the strain may be due both to external causes (external forces) and to the presence of texture defects in the continuum, described according to the treatment introduced by Volterra at the beginning of the 20th century [四]. Extending this view to space-time we expect curvature to be originated both by matter/energy and by Volterra-like texture defects.

\section{Dark energy}

We may now try to use the Lagrangian density of eq. 3.3 in order to describe the evolution of the universe on a large scale. The first move is to introduce the Robertson-Walker symmetry that we recognize in the cosmos. Though natural and in a sense obvious, the homogeneity and isotropy of space in the universe (at a large enough scale) is not a necessary consequence of the matter/energy distribution. So let us assume that the symmetry is caused by the presence of a cosmic defect in the four-dimensional manifold. The primordial ${ }^{1}$ defect produces a built in strain of space time; then the matter/energy distribution consistently responds to the global symmetry of the manifold.

The detailed calculations have been made in ref. [目] and the result is quite interesting. The additional 'elastic' terms in the Lagrangian density are responsible for an accelerated expansion, thus providing a quite natural interpretation for the dark energy. The Hubble parameter as a function of the redshift parameter $z$ turns out to be (using the standard relativistic cosmology notation):

$$
H=\frac{\dot{a}}{a}=c\left\{\frac{\kappa}{3}\left(1+z^{3}\right)\left[\rho_{m 0} c^{2}+\rho_{r 0}(1+z)\right]-\frac{B}{4}\left(1+\frac{(1+z)^{2}}{a_{0}^{2}}\right)^{2}\right\}^{1 / 2}
$$

In the equation, $\rho_{m 0}$ is the present average matter density of the universe and $\rho_{r 0}$ the present radiation energy density; $a_{0}$ is the present scale factor. It is also

$$
B=\mu \frac{2 \lambda+\mu}{\lambda+2 \mu}
$$

\footnotetext{
${ }^{1}$ Every reference to time is due to our $3+1$ attitude, but in a full four-dimensional view 'before' and 'after' are just conventions: everything 'is there', including the defect.
} 
The situation becomes even more transparent writing the effective equation of state of the 'strain fluid' corresponding to the deformation energy density:

$$
w=\frac{p}{\rho c^{2}}=-\frac{3 a^{4}+2 a^{2}-1}{3\left(a^{2}+1\right)^{2}}
$$

It is easily seen that for $a \rightarrow 0$, i.e. close to the initial singularity (here the topological defect fixing the global symmetry) it is $w \rightarrow 1 / 3$; for $a \rightarrow \infty$ it is $w \rightarrow-1$. In other words, at the beginning the 'strain fluid' behaves like radiation and the universe undergoes a slowing down expansion; in late cosmic times it behaves like the cosmological constant and the expansion is accelerated.

It is possible to optimize the parameters appearing in eq. 4. lin order to comply with a number of cosmological tests [[5]. The value obtained for $B$ is $(2.28 \pm 0.08) 10^{-52} \mathrm{~m}^{-2}$ [目].

The cosmological tests on which the present theory has been verified have been: the luminosity curves of SnIa supernovae; the primordial nucleosynthesis (correct proportion between hydrogen, deuterium and helium); CMB acoustic horizon; Barion Acoustic Oscillations; structure formation after the recombination era. The agreement and the consistency are as good as for the standard $\Lambda \mathrm{CDM}$ theory, but in this case an intuitive and reasonably simple physical interpretation of what happens is at hands.

\section{Smaller scales and different symmetries}

Considering the order of magnitude of $B \sim \lambda \sim \mu$ we do not expect effects from the space-time strain to show up at the scale, say, of the solar system. It is however interesting and useful to try and explore the effect of the presence of a classical energy density of the vacuum combined with a local spherical symmetry in space (cylindrical in space-time) or chiral symmetry in space-time; the former corresponds to the Schwarzschild problem in GR, the latter to the Kerr solution or in general to steadily rotating sources of curvature.

The equations to solve are obtained introducing the mentioned symmetries in the Lagrangian density 3.3 in vacuo, i.e. outside of the matter distribution. Unfortunately we have not succeeded in finding analytic solutions, but one is forced to have recourse to approximations. An interesting feature is that, in the gravitational potential, a term appears proportional to the squared coordinate distance, $r^{2}$, from the center of the source $[\square]$. The corresponding approximated metric tensor resembles the one of de Sitter space, but for the fact that here $g_{00} \neq g_{r r}^{-1}$. Apparently the additional terms acquire importance at the scale of galaxies clusters, however the approximation does not allow for clear cut conclusions. A preliminary consideration of the space-time surrounding a rotating mass gives evidence for modifications of the metric tensor qualitatively similar to the ones for the 'Schwarzschild' case.

Considering the origin of the local symmetry, a question, similar to the one posed on the global symmetry of the universe, may be asked. Is the local symmetry a self-consistent consequence of the matter distribution or can it originate from the presence of a local texture defect? In fact, at least in the case of spherical space symmetry, it is possible to find solutions, though approximate [ $[$ ] , preserving (and producing) the symmetry even in the absence of matter and starting from the very center of the reference frame. 


\section{Is this 'massive' gravity?}

Looking at the 'elastic' terms in 3.3 it is immediate to find a strong resemblance (if not a coincidence) with the Fierz and Pauli (FP) massive gravity Lagrangian [ [ $]$ ] that I am reproducing here for convenience:

$$
\mathscr{L}_{F P}=\frac{m^{2}}{4}\left(h^{2}-h_{\alpha \beta} h^{\beta \alpha}\right)
$$

Now the $h_{\mu v}$ tensor is the first order (linearized) difference between the actual and the Minkowski metric tensor, $\eta_{\mu \nu}$.

The visible difference is in that the Fierz and Pauli Lagrangian density contains just one parameter, $m$, interpreted as a mass, whereas the SST has two: $\lambda$ and $\mu$, read as the Lamé coefficients of space-time. In order to formally reduce FP massive gravity to SST it should be $\lambda=-2 \mu=m^{2}$. It is well known that the massive gravity Lagrangian suffers of various inconveniences and in particular it is associated with ghosts. It is then important to evidence the main and deep difference between SST and massive gravity: the latter has an approximate Lagrangian where it is assumed that the $h$ 's are much smaller than the corresponding elements of the Minkowski metric tensor; this is not the case of SST, where the strain tensor needs not be small with respect to the reference Euclidean metric and is assumed to be exact.

Various recent evolutions of the initial FP massive gravity [Q] accentuate the difference with the SST, introducing an additional auxiliary metric $f_{\mu \nu}$, used to build a $H_{\mu \nu}$ tensor allowing for higher order terms in the Lagrangian. By that approach massive gravity becomes an effective bimetric theory. SST, viceversa, is no bi-metric theory, because the only dynamical variables are the elements of the unique physical metric tensor $g_{\mu \nu}$ or (which is equivalent) of the strain tensor, since the Euclidean reference metric is fixed.

\section{Conclusion}

We have seen that, introducing into the Lagrangian density of space-time an elastic deformation energy density of the manifold, it is possible to account for the observed accelerated expansion of the universe and in the same time to allow consistently for a number of important cosmological tests. At smaller than cosmological scale the strain energy can also play an important role; an open interesting possibility is that local symmetries could be originated by the presence of defects, just as it happens at the cosmic scale. In analogy with the three-dimensional elasticity theory, the outlined SST could be an emergent theory stemming out of microscopic interactions, but there one has to face the well known problem of the mutual incompatibility between the GR approach and quantum mechanics and, even more, the problem of the duality between space-time, on one side, and matter/energy, on the other. Summing up, the SST is a promising approach to the description of space-time at a big enough scale and deserves attention and further investigations.

\section{References}

[1] A. Tartaglia, 'The Strained State Cosmology', in Aspects of Today's Cosmology, Ed. A. Antonio-Faus, InTech, Rijeka, (2011) 30-48.

[2] A. Tartaglia and N. Radicella, Class. Quantum Grav., Vol. 27 (2010) 035001. 
[3] A. Tartaglia, arXiv:1207.0626v1 (2012)

[4] V. Volterra, Ann. Sci. de l'École Normale Supérieure, Vol. 24, (1904) 401-517.

[5] N. Radicella, M. Sereno and A. Tartaglia, Int. J. Mod. Phys. D, Vol. 20, No. 6 (2011) 10391051.

[6] N. Radicella, M. Sereno and A. Tartaglia, MNRAS, Vol. 429 (2013), 11491155.

[7] N. Radicella, M. Sereno and A. Tartaglia, Class. Quantum Grav., Vol. 29 (2012) 115003.

[8] M. Fierz and W. Pauli, Proc. Roy. Soc. Lond., Vol. A173 (1939) 211-232.

[9] C. de Rahm, G. Gabadadze, Phys. Rev. D, Vol. 82 (2010) 044020. 